\title{
On DESTINY Science Instrument Electrical and Electronics Subsystem Framework
}

\author{
Semion Kizhner and Dominic J. Benford \\ National Aeronautics and Space Administration \\ Goddard Space Flight Center \\ Greenbelt Road, Greenbelt MD, 20771 \\ 301-286-1294 \\ Semion.Kizhner-1@nasa.gov \\ Tod R. Lauer \\ National Optical Astronomy Observatory \\ lauer@noao.edu
}

Abstract-Future space missions are going to require large focal planes with many sensing arrays and hundreds of millions of pixels all read out at high data rates ${ }^{1,2}$. This will place unique demands on the electrical and electronics (EE) subsystem design and it will be critically important to have high technology readiness level (TRL) EE concepts ready to support such missions. One such mission is the Joint Dark Energy Mission (JDEM) charged with making precise measurements of the expansion rate of the universe to reveal vital clues about the nature of dark energy - a hypothetical form of energy that permeates all of space and tends to increase the rate of the expansion. One of three JDEM concept studies - the Dark Energy Space Telescope (DESTINY) was conducted in 2008 at the NASA's Goddard Space Flight Center (GSFC) in Greenbelt, Maryland. This paper presents the EE subsystem framework, which evolved from the DESTINY science instrument study. It describes the main challenges and implementation concepts related to the design of an EE subsystem featuring multiple focal planes populated with dozens of large arrays and millions of pixels. The focal planes are passively cooled to cryogenic temperatures (below $140 \mathrm{~K}$ ). The sensor mosaic is controlled by a large number of Readout Integrated Circuits and Application Specific Integrated Circuits - the ROICs/ASICs in near proximity to their sensor focal planes. The ASICs, in turn, are serviced by a set of "warm" EE subsystem boxes performing Field Programmable Gate Array (FPGA) based digital signal processing (DSP) computations of complex algorithms, such as sampling-upthe-ramp algorithm (SUTR), over large volumes of fast data streams. The SUTR boxes are supported by the Instrument Control/Command and Data Handling box (ICDH Primary and Backup boxes) for lossless data compression, command and low volume telemetry handling, power conversion and for communications with the spacecraft. The paper outlines how the JDEM DESTINY concept instrument EE subsystem can be built now, a design, which is generally

\footnotetext{
${ }^{1}$ U.S. Government work not protected by U.S. copyright

${ }^{2}$ IEEEAC paper \# 1429, Version 4, Updated October 19, 2009
}

applicable to a wide variety of missions using large focal planes with large mosaics of sensors.

\section{TABLE OF ConTents}

\subsection{INTRODUCTION}

2.0 KEY DRIVING REQUIREMENTS

3.0 DESTINY INSTRUMENT EE SUBSYSTEM CONCEPT

4.0 HERITAGE AND KNOW-HOW AT NASA GSFC

CONCLUSIONS

ACKNOWLEDGEMENTS

REFERENCES

BIOGRAPHY

\section{INTRODUCTION}

In this paper we present the DESTINY concept for the EE subsystem. The EE subsystem is controlling a few large focal planes populated with a mosaic of homogeneous sensors and readout ASICs. The instrument electronics are primarily tasked with the readout and control of a large array of large format detector assemblies (mosaic of $\mathrm{HgCdTe}$ Astronomy Wide Area Infrared Imager with $2 \mathrm{~K} \times 2 \mathrm{~K}$ resolution or $\mathrm{H} 2 \mathrm{RG}$ sensors) in the science focal plane. The science focal plane is passively cooled to a cryogenic temperature. The science array is sampled (nondestructive sampling allowed by the H2RGs) multiple times within an exposure. The focal plane electronics feature a fast readout and onboard signal and data processing of hundreds of frames within an exposure using large external memories, followed by additional lossless data compression in the ICDH box and formatting the data prior to its transmission to the spacecraft Solid-State Recorder (SSR).

For the DESTINY instrument EE subsystem the principal challenges are the large number of science detector elements (pixels) - $362 \mathrm{~K} \times 2 \mathrm{~K}$ H2RG sensors $\left(1.512 \times 10^{8}\right.$ pixels, where $\left.\mathrm{K}=1024\right)$ and the short time in which these elements must be read out $(1.31072 \mathrm{~s})$ on multiple (32) $100 \mathrm{KHz}$ channels $(0.1152 \mathrm{Gps})$ and have their data processed and stored $\left(<<10^{2} \mathrm{~s}\right)$, and the resulting 
frame sensor and readout noise level of 6e- (as summarized in Section 2). These require readout by a sensor dedicated ASIC that is retrieving and enhancing sensor frame signal, digitizing the signal and controlling the sensor chip assemble (SCA) - such as the ASIC from Teledyne called SIDECAR. The 36 SIDECAR ASICs and their built-in 36 microprocessors perform the signal digitization at 16 bits, resulting in order of magnitude larger volumes of digital data bits. It transmits the digital data to warm electronics that ingest and process exposure science data, and transmit a single resulting exposure frame (for each sensor) to the spacecraft. The fast processing of such large volumes (1.85Gbs) of data using advanced algorithms, such as SUTR based on the up-the-ramp algorithm by Offenberg, Fixsen and John Mather, is at the core of this challenge. The EE subsystem is processing from $277.5 \mathrm{~Gb}$ to $1665 \mathrm{~Gb}$ for 150 s and 900 s exposures correspondently and outputs 0.34 $\mathrm{Gb}$ of data for on-board recording, resulting in SUTR data compression by a factor $\mathrm{L}$, where $113<=\mathrm{L}<=670$ for exposures of 150 to 900 seconds. This compression factor is the number of frames from which one frame is generated for output as a result of an exposure data processing. The onboard data processing must sustain the 150 s integrations assuming an average $86 \%$ observing efficiency over a day. SUTR also reduces the sensor and readout noise by a factor proportional to sqrt(L) or between 10 to 25 times, resulting in readout noise reduction to required $6 \mathrm{e}-$ level.

The solution to the DESTINY-type instrument requirements of intensive data processing for reducing sensor and readout noise to $6 \mathrm{e}$ - and output data rate commensurate with the state-of-the-art space-to-ground communications link is both technically and fiscally feasible. The EE subsystem does not require new technology development prior to the design phase. It leverages the HST, JWST, Spitzer, GLAST, SDO, LOLA and GPM missions' EE technologies (presented in Section 4) and heritage in Science Sensors, ASICs and ROICs, onboard data processing by using reconfigurable computing (RC), FPGAs, Network and Instrument Sensor Web (ISW) [7] topologies, fine thermal control sensors, high speed data interfaces (Spacewire, High-Speed Low-Voltage Differential Signaling or LVDS), harness radiation protection, and large 3.6 GB External Synchronous dynamic random access memory (SDRAM) augmented by a $0.34 \mathrm{~GB}$ SDRAM Buffer for exposure frames output to the ICDH. The SUTR maps the 3.6 GB SDRAM into 0.34 GB memory buffer for each exposure for all 36 sensors, which in-turn is compressed conservatively at $2: 1$ ratio by the $\mathrm{ICDH}$ for output of $170 \mathrm{MB}$ per exposure to the SC SSR for the shortest exposure of 150 seconds.

The science focal plane's large sensors with readout electronics are of proven technology for small mosaic configurations. The science sensors are controlled individually by high-performance ASICs. Each ASIC ingests sensor analog signals and outputs 16-bit digital data on $416-\mathrm{MHz}$ channels to powerful processing boards (called "SUTR Boards") with built-in redundancy. The processing boards' FPGA cores ingest the ASIC output digital data, perform the SUTR data processing and output an exposure frame for each sensor for different science exposure mode integration times (150 seconds to 900 seconds) from its exposure buffer to the ICDH. The instrument flight software running on the RAD750 computer in the ICDH controls the data processing. A SUTR board comprises two large FPGAs (80 FPGAs total) running the pre-processing and SUTR data processing (the instrument flight software application computationally intensive functions) in firmware and hardware and supported by a $125 \mathrm{MB}$ SDRAM module on each SUTR board. The output of a processing board digital module is a sequence of packetized sensor frames comprising an operational science mode exposure. These packets are networked over Spacewire interfaces to the ICDH for 2:1 lossless compression and the Consultative Committee for Space Data Systems (CCSDS) $2 \%$ overhead packetization, and forwarded by the ICDH to the Spacecraft SSR over high speed Spacewire interface. This results in a maximum $170 \mathrm{MB}$ exposure frames data stream transmitted to the SC SSR for each exposure. For 150 s exposures and $86 \%$ observing efficiency this amounts to 864 daily frames for each of the 36 sensors or $1.5 \mathrm{~Tb}$ that determines the size of the SC SSR

The ASIC/ROICs selection of SIDECAR ASIC and its configuration architecture, as well as SUTR processing and reference pixels utilization for noise reduction, address the sensor readout noise requirements of $6 \mathrm{e}$ - per exposure. The implementation of the instrument flight software for the data processing in the FPGA hardware addresses the data volumes, rates and onboard computational complexity requirements. The hardware compression modules card architecture (assisted by RAD750 and the instrument flight software in the ICDH) implements the required throughput requirements to the spacecraft SSR. All critical hardware components are radiation hardened.

The flow of analog signal and digital data is organized in packets identifying the data source, the frame boundaries, the frame unit of information - line beginning and end codes for analog signals and heritage packet encapsulation for digital data.

The harness between cryogenic sensors and SIDECAR ASICs and the EE subsystem boxes comprises thousands of conductors. However, such harness design is quite mature, but there are to be tradeoffs of electrical/thermal issues for the SCA-ASIC harness.

The JDEM DESTINY concepts were developed over the last few years and described in [1-7].

The DESTINY EE subsystem concept and framework were developed to the DESTINY instrument top-level and key driving requirements. The following Section 2 Key Driving Electronics Requirements depicts these requirements. The EE subsystem concept, framework and architecture, which meet these challenges and requirements, are presented in Section 3. Section 4 depicts relevant experience and facilities at NASA GSFC to support the JDEM DESTINY-type instrument development. 


\section{KEY DRIVING ELECTRONICS REQUIREMENTS}

Weak Lensing (WL), Baryon Acoustic Oscillations (BAO) and Super Nova (SN) measurements are the known techniques to probe dark matter and energy. The Joined Dark Energy Mission Near Infrared Instrument (NIRI) employs all three techniques.

Although many JDEM requirements are still in the processes of change, evolution and refinement, some are known and mature at this time and sufficient to develop the EE subsystem concept and architecture. Among these are:

- Observations Probe of Faint Super Nova Sources in Near Infrared (NIR) Band

- Probe for Baryon Oscillations

- Probe for Weak Lensing

- Precise Pointing and distant-in-time RePointing within Milli-arc Seconds

- Fine Guidance System (FGS) with FGS Sensors in Observatory Telescope Boresight Focal Plane

- FGS Sensors readout by the EE subsystem and on-board processing within the EE subsystem or Spacecraft Attitude Control System (ACS) or Both

- EE subsystem Signal and Data Processing on an FGS Control Pulse upon Pointing-for-Exposure Completion

- Large Science Focal Plane and Sensor/ASIC Mosaic Control

- Use of SIDECAR ASIC in Focal Plane Electronics (FPE) (cold electronics)

- Reduce on-board data volume

- SUTR data processing resulting in compression better than 150:1, followed by

- Data compression in ICDH at 2:1 ratio

- Reduce on-board Sensor/Readout noise and reject cosmic rays

- SUTR processing in Image Frame Processors within the Off-Focal Plane Electronics in the EE subsystem (warm electronics)

- Cosmic Ray Rejection is an Integral Part of Noise Reduction in SUTR processing

- Science Sensor Readout on maximum number of channels to reduce frame clock-out rate and reduce readout noise
- Maintain Focal Plane and cold electronics at stable cryogenic temperatures

- Maintain Focal Plane and cold electronics at stable cryogenic temperatures

- Active thermal control electronics by ICDH

- Appropriate harness materials to minimize

parasitic heat loads

- Detectors cutoff is at $\sim 1.7 \mu \mathrm{m}$

-This allows the detector to operate at relatively warm temperatures $(\sim-120 \mathrm{C})$ with acceptable dark current. This simplifies the instrument by allowing the use of thermoelectric cooling systems instead of the heritage cryogens or mechanical cryogenics coolers that are typical in other NIR instruments and thermal control by the EE subsystem ICDH

- Operate reliably at the Lagrange 2 Point (L2) environment for three years

- Thorough modeling of L2 radiation environment

- Use of proven radiation hardened Electrical, Electronic, and Electromechanical (EEE) parts - Use of radiation mitigation schemes (spot shielding, redundancy, etc.) where necessary

- High performance on-board data processing - FPGA-based hardware processing in Image Frame Processors

- HyperX Experiment on MISSE-7, carrying super-computing hardware

- Proven RAD750 general-purpose processor in ICDH

- Dedicated image compression ASICs in ICDH at 2:1 ratio and data formatting for storage in SSR

- Warm electronics are housed in spacecraft bay -Ambient temperature

- The FGS carries its own star catalog of some 3000 stars, each describes by 5 double-precision floating point numbers and requiring 120,000 bytes memory. It must be upgradable from the ground.

- All on-board processing within the EE subsystem is synchronized with an FGS Timing Control Pulse of Completion of Precise Pointing for an observation exposure. If FGS is part of the SC ACS there must be a timing control pulse interface from SC to the EE Subsystem.

- There must be an option for real-time downlink - Critical science telemetry subset bypassing the SC SSR for SSR function redundancy. 
- For a single string design the SC SSR must be radiation hardened

$\bullet$ Radhard SDRAM-based boards. With $51.5 \mathrm{~Gb}$ modules per board the SSR is populated with some 100 boards and weighing more than $50 \mathrm{~kg}$.

The following Figure 1 depicts the EE Subsystem concept framework and architecture tailored to the key requirements.

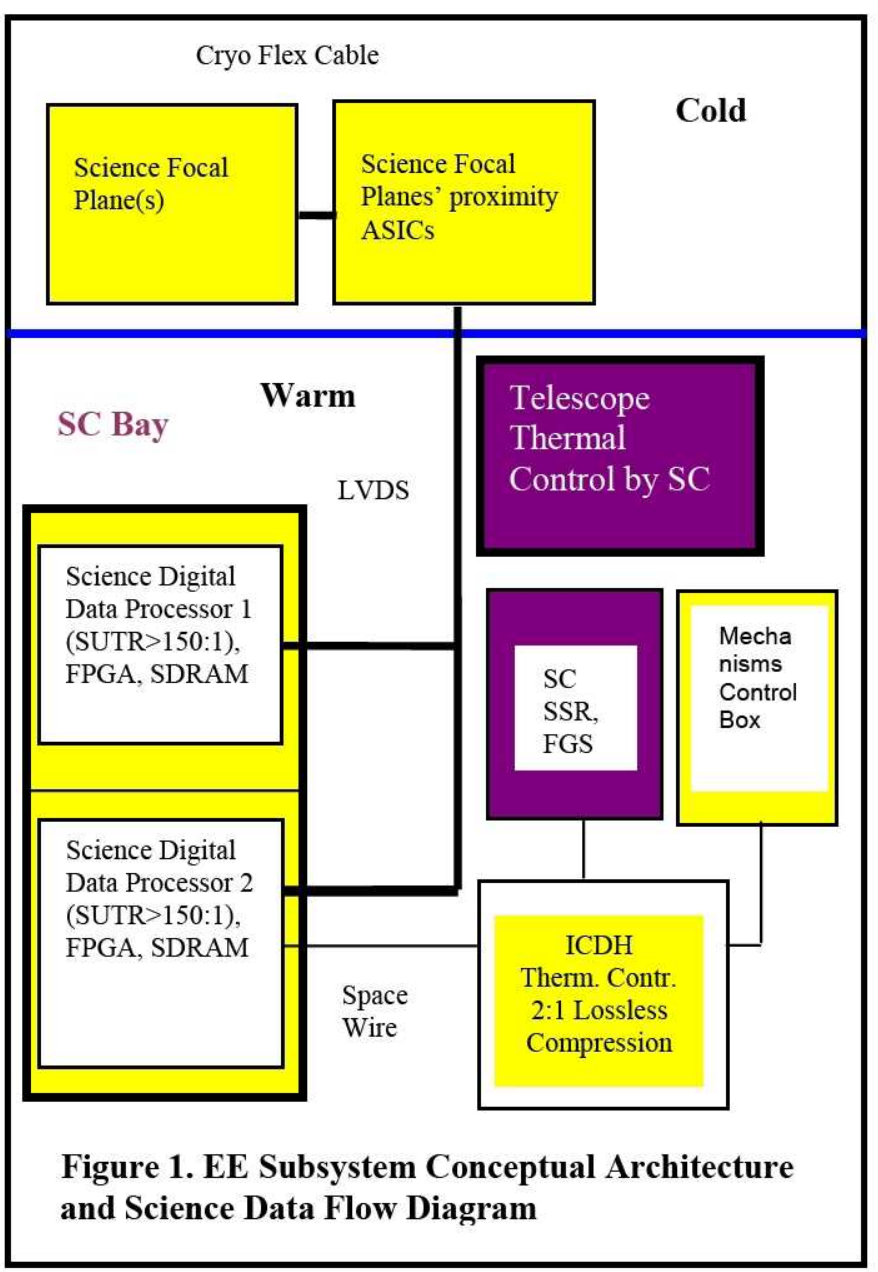

\section{DESTINY INSTRUMENT EE SUBSYSTEM CONCEPT}

The EE subsystem signal and data flow are outlined in the diagram in Figure 1. The instrument EE subsystem components are presented in yellow.

The DESTINY EE subsystem concept was developed on electronics board functionality level and $\mathrm{EE}$ box level. EE subsystem data processing box (SUTR box with a board dedicated to each sensor/ROIC pair) comprises a number of boards, for example a 12 -board box, resulting in a number of SUTR boxes depending on the actual size of focal plane.
The DESTINY EE subsystem scope is similar, but at the same time very different, from heritage instruments. It encompasses all the heritage subsystems' electrical and electronics field, such as providing power to all other DESTINY payload subsystems (except power for telescope thermal control circuits and payload survival heaters), control of the mechanisms and thermal control of focal planes and all other subsystems where power dissipation is low. Thermal control of the telescope, for example, that involves large power dissipation, is delegated to the spacecraft.

The novel function of the DESTINY EE subsystem is readout of large mosaics of large sensors and the on-board processing application requiring supercomputer resources. For that the EE subsystems provides a platform for the Instrument Flight Software Data System that is distributed between the ICDH (Heritage Operating System and Command and Telemetry high-level processing function), as well as new functions of developing the instrument application algorithms for their further programming by hardware engineers on the FPGAs and DSPs which provide the super-computer capabilities. The readout electronics ASICs comprise multiple microprocessors requiring its own programming. We envision that this programming also partially falls into the domain of the instrument flight software. This is a shift in paradigm from heritage flight software and it requires carful considerations in the conceptual design of the newer instrument flight software, such as for DESTINY. Some of he EE subsystem functions, for example those related to mechanisms and thermal control, may be developed by the thermal and mechanical subsystem team. However, the EE subsystem is a placeholder for these functions final deliverables.

\section{IntRoduction The Heritage AND KNOW- How AT NASA GSFC}

The EE subsystem key technologies, the H2RG/SIDECAR sensor/ROIC, the ACTEL RTAX-2000S FPGAs, the 1Gb SDRAM Memory Modules and the RAD750 Computer and Harness were all used in previous flight missions, and sensors and ASICS with their interconnecting flex cables at cryogenic temperatures, as depicted in the following displays. The usage of abbreviations for missions in Section 4 can be readily interpreted from a search engine on the Internet. The few used more than once in the paper are:

COBE - Cosmic Background Explorer

MAP - Microwave Anisotropy Probe

JWST - James Web Space Telescope

SDO - Solar Dynamics Observatory

LRO - Lunar Reconnaissance Orbiter

HST-SM4 - Hubble Space Telescope Servicing Mission 4

RNS - Relative Navigation System

GPM - Global Precipitation Mission

MISSE-7 - Materials on International Space Station Experiment. 
Cryogenics Control Electronics (including Cabling and Harnessing) and Cryogenic Temperature Passive Control

-Related Experience: COBE, MAP, ASTRO-E, JWST

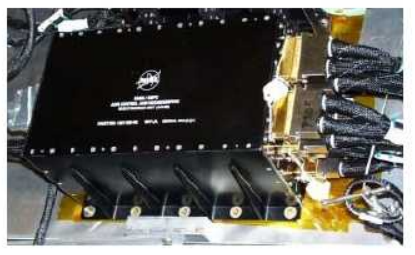

ASTRO-E2 XRS Detector with Cryo Cables, and ADR Control Electronics

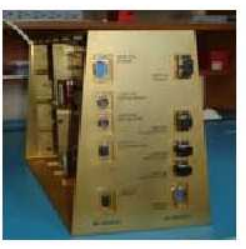

JWST NIRSpec Focal Plane Electronics

\section{FPGA Flight Design and Application}

-Related Experience: LRO/LOLA, HST-SM4 RNS

-GSFC jointly sponsors the annual Military and Aerospace Programmable Logic Device (MAPLD) Conference

-GSFC lead an Actel RTAX FPGA risk reduction study for NESC

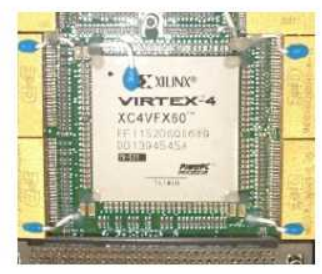

Spacecube Virtex-4

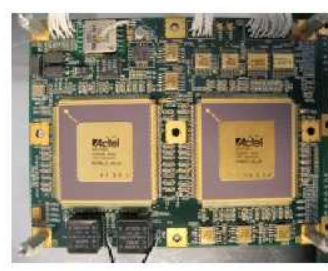

LRO Actel RTAX-2000S

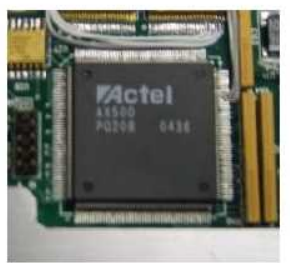

LOLA Actel RTAX-250S

High Density Memory Devices

-Related Experience: LRO, SDO, HST-SM4 RNS

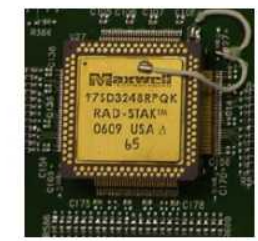

1.5Gb SDRAM (SDO Bulk Memorv)
World leadership and expertise in FPGA's, radiation effects, and EEE parts:

-Related Experience: Numerous In-House and Out-ofHouse Missions

-EEE-INST-002 has become the industry standard reference for testing/qualifying EEE parts

-Acknowledged experts in assessment and evaluation of single event effects (both digital and analog) and dose effects on analog, optoelectronic and fiber optics

-GSFC jointly sponsors the annual Nuclear \& Space Radiation Effects Conference (NIRSpec).

\section{Advanced Packaging Concepts}

-Related Experience: SWIFT-BAT, MLA Messenger,

Mars Science Orbiter

\section{H2RG Detector/SIDECAR ASIC}

-Related Experience: JWST, HST-ACS
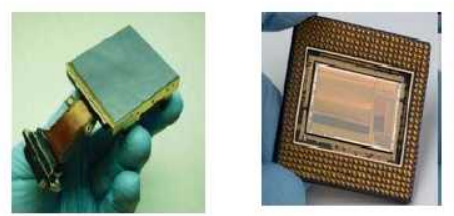

H2RG Detector with Cryo Flex Cable/SIDECAR ASIC from

Teledvne

\section{RAD750 Processor Board}

-Related Experience: LRO, SDO, GPM

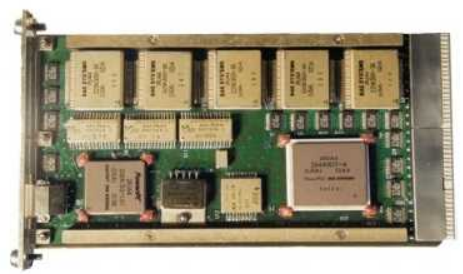

RAD750 Single Board Computer

On-board Digital Signal Processing and Compression -Related Experience: ASTRO-E, ICESAT/GLAS, HST-SM4 RNS Spacecube, MMS, and MISSE-7

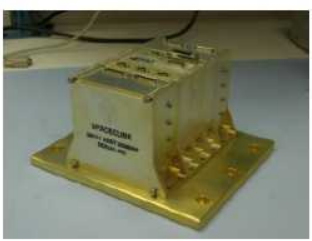

HST-SM4 RNS Spacecube

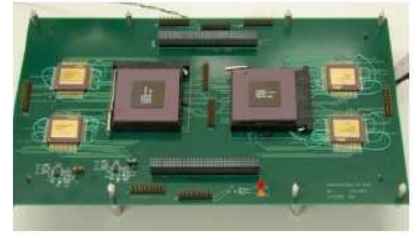

MMS

Data Compression ASICs 


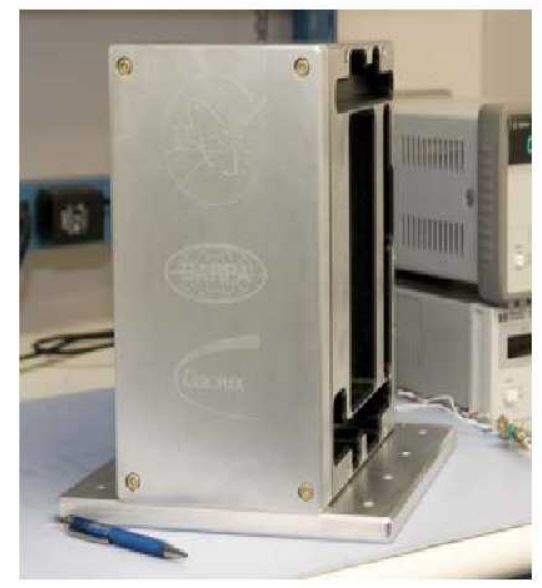

MISSE-7 HyperX Experiment Enclosure. HyperX is an ISS-bound ELC experiment delivered to ISS by STS-129.

\section{Operating Reliably in the L2 Environment for three years}

-Related Experience: MAP, JWST

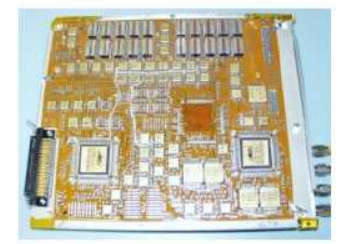

WMAP C\&DH Processor Board

\section{Conclusions}

We have introduced the JDEM DESTINY-type instrument EE subsystem challenges, presented its key driving electronics requirements and the EE subsystem concept framework and science data flow diagram. In the Heritage and Know-How Section 4 we elaborated on the NASA Goddard Space Flight Center experience and capabilities related to the development of such an instrument using the available high TRL key technologies. The NASA Goddard Space Flight Center has the end-to-end flight electronics design/fabrication/testing capabilities: Circuit and Chassis Design, Layout, Fabrication and Assembly, Electrical and Environmental Test Facilities, as well as Established Set of Standards, Requirements and Procedures for the development of the instrument now. We have presented the concept of an EE subsystem and a framework that are generally applicable to a wide range of future missions needing low cost and high reliability EE subsystem implementation that enables large mosaics of sensing arrays with 100 's of millions of pixels operating at cryogenic temperatures.

\section{ACKNOWLEDGEMENTS}

The authors would like to acknowledge and thank the following members of the Goddard Space Flight Center: Robert L. Kasa, John F. McCabe, Maxime Pinchinat, Wesley A. Powel and Richard B. Katz for compiling the technology pictures. Special thanks are to Clifton E. Jackson and James M. Lohr for constructive review of the paper. We also acknowledge and thank Michael Johnson of GSFC of providing the HyperX picture.

\section{REFERENCES}

[1]. The DESTINY concept for the Joint Dark Energy Mission (JDEM). Morse, J. A., Lauer, T. R., \& Woodruff, R. A. (2004), Proceedings of the SPIE, 5487, 1484

[2]. Technical Implementation of the DESTINY Mission Concept. Woodruff, R. A., Morse, J. A., \& Lauer, T. R. 2004, Proceedings of the SPIE, 5487, 1545

[3]. DESTINY: The Dark Energy Space Telescope. Lauer, T. R., \& Destiny Science Team (2005), ASP Conf. Ser. 339: Observing Dark Energy, 339, 79

[4]. DESTINY: The Dark Energy Space Telescope. Lauer, T. R. (2005), New Astronomy Review, 49, 354

[5]. Destiny: A Candidate Architecture for the Joint Dark Energy Mission. Benford, D. J., \& Lauer, T. R. (2006), Proceedings of the SPIE, 6265, 671

[6]. Simulations of Sample-Up-The-Ramp for Space-Based Observation of Faint Sources. Dominic J. Benford, Tod R. Lauer, D. Brent Mott 2008, Proc. Of SPIE Vol. 7021, 70211V-1 (2008)

[7] On Representative Spaceflight Instrument and Associated Sensor Web Framework. Semion Kizhner, Dr. Umeshkumar Patel, Wesley A. Powell and Meg Vootukuru, Proc. of IEEE Aerospace Conference (2007). 


\section{BIOGRAPHY}

Semion Kizhner is an aerospace engineer with the National Aeronautics and Space Administration at the Goddard Space Flight Center. He proposed the development of the Hilbert-Huang Transform Data Processing System and has been leading the HHT-DPS development team. He participated recently in evaluation of the NASA Advanced Space Technology proposals. He published a dozen of technical papers and mentored numerous undergraduate, graduate and doctoral students in the NASA Education Programs. He graduated from Johns Hopkins University with an MS degree in computer science.

Dr. Benford joined the Goddard Space Flight Center in Greenbelt, Maryland in 2001. Prior to this, he spent over two years in the Infrared Astrophysics Branch at Goddard, first as an NRC Research Associate, then as a Principle Physics Engineer for Raytheon/ITSS. He pursued research in far-infrared detector development as a Fellow of the GSRP program at Goddard from 1994-1998. He has pursued research in continuum detectors for ground-based submillimeter observations, near-Terahertz heterodyne receiver technology, submillimeter observations of star forming regions and ultraluminous galaxies, and cryogenic systems for space observatories. He has led and participated in several mission concept studies, including the SAFIR Vision Mission and, currently, the Destiny mission to characterize dark energy. His current primary research focus is in the development of ultrasensitive bolometers for farinfrared and submillimeter astronomy and supporting technologies. He holds a Ph.D., Physics, California Institute of Technology (1999).

Dr. Tod R. Lauer is an astronomer on the research staff of the National Optical Astronomy Observatory. He was a member of the Hubble Space Telescope Wide Field and Planetary Camera team, and is a founding member of the Nuker Team. His research interests includes observational searches for massive black holes in the centers of galaxies, the structure of elliptical galaxies, stellar populations, large scale structure of the Universe, and astronomical image processing. He was recently the PI of the Destiny JDEM concept study. Asteroid 3135 Lauer is named for him. He holds a Ph.D. in Astronomy from the University of California, Santa Cruz (1983). 